$>$ Les virus à ARN sont responsables de maladies graves telles que la grippe, la dengue, I'hépatite C, la rougeole ou la bronchiolite des nourrissons. Ils représentent également une menace émergente en raison des échanges internationaux et des bouleversements écologiques liés aux activités humaines. Notre compréhension des mécanismes cellulaires qui contrôlent la réplication virale a fortement progressé ces dernières années, et il apparaît que des composés capables de cibler la cellule hôte, plutôt que le virus luimême, pourraient inhiber un large éventail de virus à ARN. Ainsi, différents laboratoires privés et universitaires sont en quête de molécules stimulant la réponse antivirale innée des cellules hôtes. Une stratégie intéressante consiste à rechercher des molécules induisant l'expression des ISG (interferon-stimulated genes), lesquels constituent un vaste ensemble de gènes antiviraux activés en particulier par les interférons de type I. Pour atteindre cet objectif, nous avons mis au point un test phénotypique basé sur des cellules humaines transfectées avec un gène rapporteur luciférase sous contrôle d'un élément de réponse aux interférons (ISRE). Ce système a été utilisé pour cribler une chimiothèque d'environ 54000 composés. Parmi les molécules sélectionnées, nous avons montré que le composé DD264 stimule la réponse immunitaire innée dans les cultures cellulaires, et présente une activité antivirale à large spectre. En recherchant la cible de ce composé, nous avons montré qu'il inhibe la voie de biosynthèse de novo des pyrimidines, et notamment la quatrième enzyme de cette voie métabolique, la dihydroorotate déshydrogénase (DHODH). Ainsi, nos résultats établissent de façon surprenante un lien fonctionnel nouveau entre la voie de biosynthèse des pyrimidines et la réponse innée antivirale. <

\section{Chémobiologie (4) \\ Activation de \\ la réponse innée \\ antivirale par \\ des inhibiteurs \\ de la biosynthèse \\ des pyrimidines}

\section{Les surprises d'un criblage phénotypique}

Pierre-Olivier Vidalain ${ }^{1}$, Marianne Lucas-Hourani ${ }^{1}$, Olivier Helynck ${ }^{2}$, Frédéric Tangy ${ }^{1}$, Hélène Munier-Lehmann²

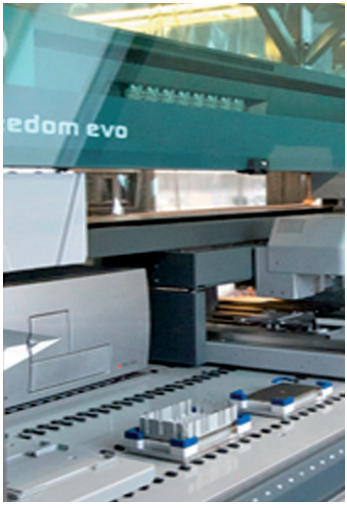

${ }^{1}$ Unité de génomique virale et vaccination, département de virologie, Institut Pasteur, CNRS UMR 3569 ,

28, rue du Docteur Roux, 75724 Paris Cedex 15, France; ${ }^{2}$ Unité de chimie et biocatalyse, département de biologie structurale et chimie Institut Pasteur, CNRS UMR3523, 28, rue du Docteur Roux, 75724 Paris Cedex 15, France. pierre-olivier.vidalain@pasteur.fr helene.munier-lehmann@pasteur.fr

Malgré des besoins importants, notre arsenal thérapeutique contre les infections virales, et notamment contre les virus à génome ARN, est extrêmement limité [1]. Si des vaccins efficaces ont été développés contre de nombreux virus mortels pour l'homme comme la variole, la fièvre jaune ou la poliomyélite, la couverture vaccinale reste bien souvent insuffisante, y compris dans les pays développés. À titre d'exemple, on assiste en France depuis quelques années à une recrudescence des cas de rougeole avec des conséquences parfois dramatiques, alors qu'un vaccin sûr et efficace existe [2]. Des molécules antivirales seraient ainsi d'une grande utilité pour traiter des patients ayant déjà déclaré l'infection. En outre, il n'existe pas de vaccin contre de nombreux virus qui représentent pourtant un problème majeur en santé publique. Par exemple, le virus respiratoire syncytial humain (HRSV) serait responsable à l'échelle mondiale d'environ 3,4 millions d'infections respiratoires sévères et d'au moins 66000 décès chez les enfants de moins de cinq ans [3]. Du fait de la multiplication des échanges internationaux, les grandes métropoles se trouvent également de plus en plus exposées à des infections tropicales d'origine

Cet article fait partie de la série «Chémobiologie » qui a débuté dans le $n^{\circ} 12$, vol. 30, décembre 2014 (www.medecinesciences.org). 


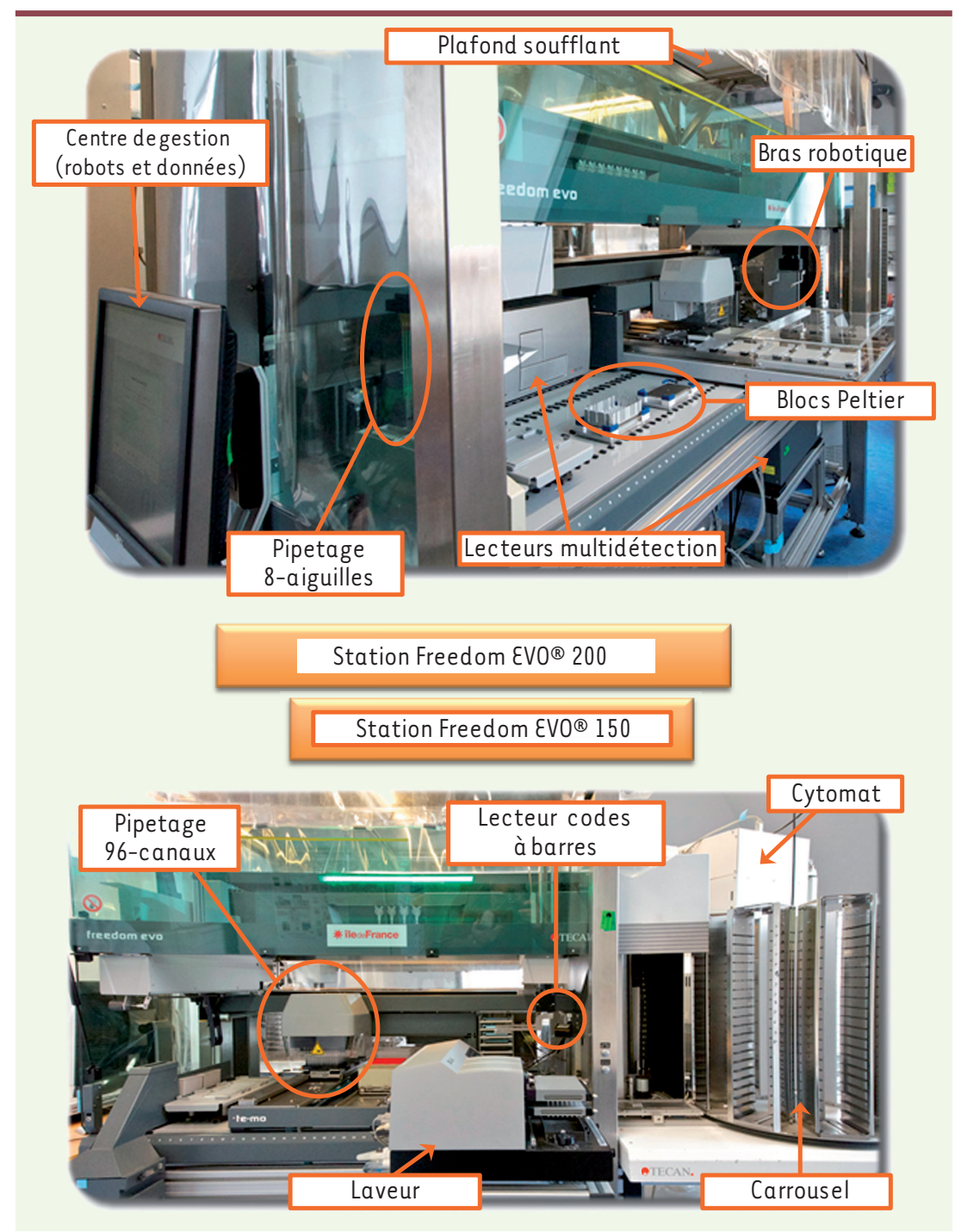

Figure 1. Descriptif de la plate-forme CBC. La plate-forme CBC a pour objectif la découverte de nouveaux outils moléculaires permettant d'étudier des structures ou des processus biologiques complexes, ou l'identification de composés à visée thérapeutique, principalement dans le domaine des anti-infectieux. Elle est opérationnelle depuis 2008 et fait partie du GDR ChemBioScreen depuis la création de ce dernier en 2011. Deux stations Freedom VVO$^{\oplus}$ (TECAN) constituent le cœur du dispositif. Elles sont placées sous des plafonds soufflants permettant de travailler en conditions stériles et sont situées dans un laboratoire de niveau de confinement 2. Chaque station contient un carrousel (température ambiante) et un incubateur (Cytomat, température régulée) où sont stockés les consommables (plaques 96 ou 384-puits et boîtes de cônes), un bras manipulateur qui va déplacer les consommables sur l'ensemble du plan de travail, deux systèmes de pipetage (96 canaux et 4 ou 8 aiguilles) permettant de réaliser des pipetages entre 1 et $250 \mu \mathrm{l}$, et un lecteur de codes à barres permettant une gestion automatisée des plaques qui entrent sur le plan de travail. Selon les applications, des cônes stériles ou des aiguilles téflonisées peuvent être utilisés pour les pipetages. L'une des stations (Freedom EVO ${ }^{\circledR} 150$, panneau inférieur) intègre un laveur de plaques spécialement adapté aux tests ELISA et aux cultures cellulaires. Sur la plus grande des deux stations (Freedom $\varepsilon V 0^{\circledR} 200$, panneau supérieur) sont intégrés deux blocs Peltier agitateurs (température réglable entre 4 et $40^{\circ} \mathrm{C}$ ) et des lecteurs multimodes de plaques permettant des mesures en absorbance, fluorescence et luminescence, ainsi que l'utilisation de la technologie AlphaScreen (Sunrise ou Infinite M200 Pro, et Infinite M1000, TECAN). Les deux stations sont pilotées par le logiciel Freedom EVOware (TECAN). Par ailleurs, les données de pipetage et de lecture sont envoyées à un serveur qui héberge un LIMS développé à façon par la société Modul-Bio, et qui permet le stockage et l'analyse de ces données ainsi que la gestion des chimiothèques.

virale, comme la dengue ou le chikungunya. Enfin, on assiste depuis quelques décennies à des phénomènes d'émergence virale liés à l'anthropisation des espaces naturels, qui facilitent la transmission des virus animaux à l'homme. L'épidémie de MERS-CoV (Middle East respiratory syndrome coronavirus) est ainsi un exemple récent d'émergence virale [4].

\section{Quelles stratégies antivirales?}

Pour mettre au point une molécule antivirale, l'approche classique consiste à cibler l'activité d'une protéine virale essentielle au cycle du virus : ARN polymérase, intégrase, hélicase ou protéase par exemple. Au milieu des années 1990, les associations d'anti- rétroviraux hautement actifs ont ainsi permis des avancées importantes dans la prise en charge des patients infectés par le VIH (virus de l'immunodéficience humaine), même s'il n'est pas encore possible d'éliminer définitivement le virus [5]. De même, des résultats révolutionnaires ont été obtenus récemment dans le traitement de l'hépatite $C$ grâce aux dernières générations d'antiviraux à action directe, qui ciblent notamment la protéase NS3-4A et la polymérase NS5B du virus de l'hépatite C (VHC) [6, 7]. Cependant, la très grande diversité des virus responsables d'infections chez l'homme ne permet pas d'envisager le développement pour chacun d'un 


\section{Origine de la chimiothèque \\ Acronyme/nom \\ Nombre de composés}

\section{Caractéristiques}

Académique; échantillon représentatif de la

Unité de gestion de la chimiothèque

nationale, CNRS UPS3035, Montpellier

CNE

640

chimiothèque nationale selon des critères de diversité maximale

Laboratoire conception, synthèse et vectorisation de biomolécules, Institut Curie, CNRS UMR176, Orsay

Centre d'études et de recherche sur le médicament de Normandie, EA 4258, Caen

Institut de chimie des substances naturelles, CNRS UPR2301, Gif-sur-yvette Curie $\quad 8560$ Académique; membre de la chimiothèque nationale

cadémique; membre de la chimiothèque nationale

Académique; membre de la chimiothèque nationale

Laboratoire de pharmacochimie de la communication moléculaire, CNRS UMR7200, faculté de pharmacie, IIIkrich

Institut de chimie et biochimie moléculaire et supramoléculaire, CNRS UMR 5246, Lyon

Unité de chimie et biocatalyse, Institut Pasteur, CNRS UMR3523, Paris

IP

Prestwick Chemical Prestwick chemical library ${ }^{\circledR}$

ChemDiv
NECAN

IK

ChemX
Académique; membre de la chimiothèque nationale

\begin{tabular}{lccl} 
ChemDiv & IK & 9360 & Commerciale ; composés prédits kinases \\
Chem-X-Infinity & ChemX & 10000 & $\begin{array}{l}\text { Commerciale ; échantillon représentatif } \\
\text { des sous-chimiothèques thématiques } \\
\text { de Chem-X-Infinity (RCPG, kinases, etc.) }\end{array}$ \\
\hline
\end{tabular}

Tableau I. Liste des différentes chimiothèques disponibles sur la plate-forme CBC.

traitement spécifique, d'où l'idée de développer des molécules à large spectre, actives contre différents virus [1]. Certaines fonctions enzymatiques - ARN polymérase, hélicase, protéase - sont suffisamment conservées entre protéines virales pour qu'il soit possible de développer de telles molécules. Récemment, un nouvel analogue de nucléoside capable d'inhiber de nombreux virus à ARN, et notamment les filovirus tels que le virus de Marburg, a été décrit et semble particulièrement efficace in vivo contre ces infections [8]. Cependant, le nombre de facteurs viraux et/ou d'activités enzymatiques ainsi conservés et qui peuvent être la cible de molécules thérapeutiques est relativement limité. Par ailleurs, la grande plasticité des génomes viraux facilite l'apparition rapide de mutants d'échappement qui rendent parfois inefficaces ce type de traitements, comme le montre l'évolution du virus de la grippe [9]. Afin de contourner ces deux obstacles, plusieurs équipes dont la nôtre cherchent à cibler la cellule hôte plutôt que le virus lui-même en bloquant des mécanismes cellulaires essentiels à la réplication virale, ou en stimulant la réponse immunitaire innée $[10,11]$.

\section{Isoler des composés antiviraux à partir de criblages biologiques et de chimiothèques}

Pour identifier des molécules ayant une activité biologique d'intérêt dans le traitement des infections microbiennes, nous avons mis en place à l'Institut Pasteur de Paris la plate-forme CBC (criblage biologique et chimiothèques). Celle-ci dispose de différents équipements permettant le criblage de composés à haut débit (Figure 1) et de plusieurs chimiothèques commerciales et académiques pour un total d'environ 57000 molécules (Tableau I). Pour isoler des composés antiviraux à large spectre à partir de ces banques, nous avons élaboré deux stratégies distinctes. Tout d'abord, nous 


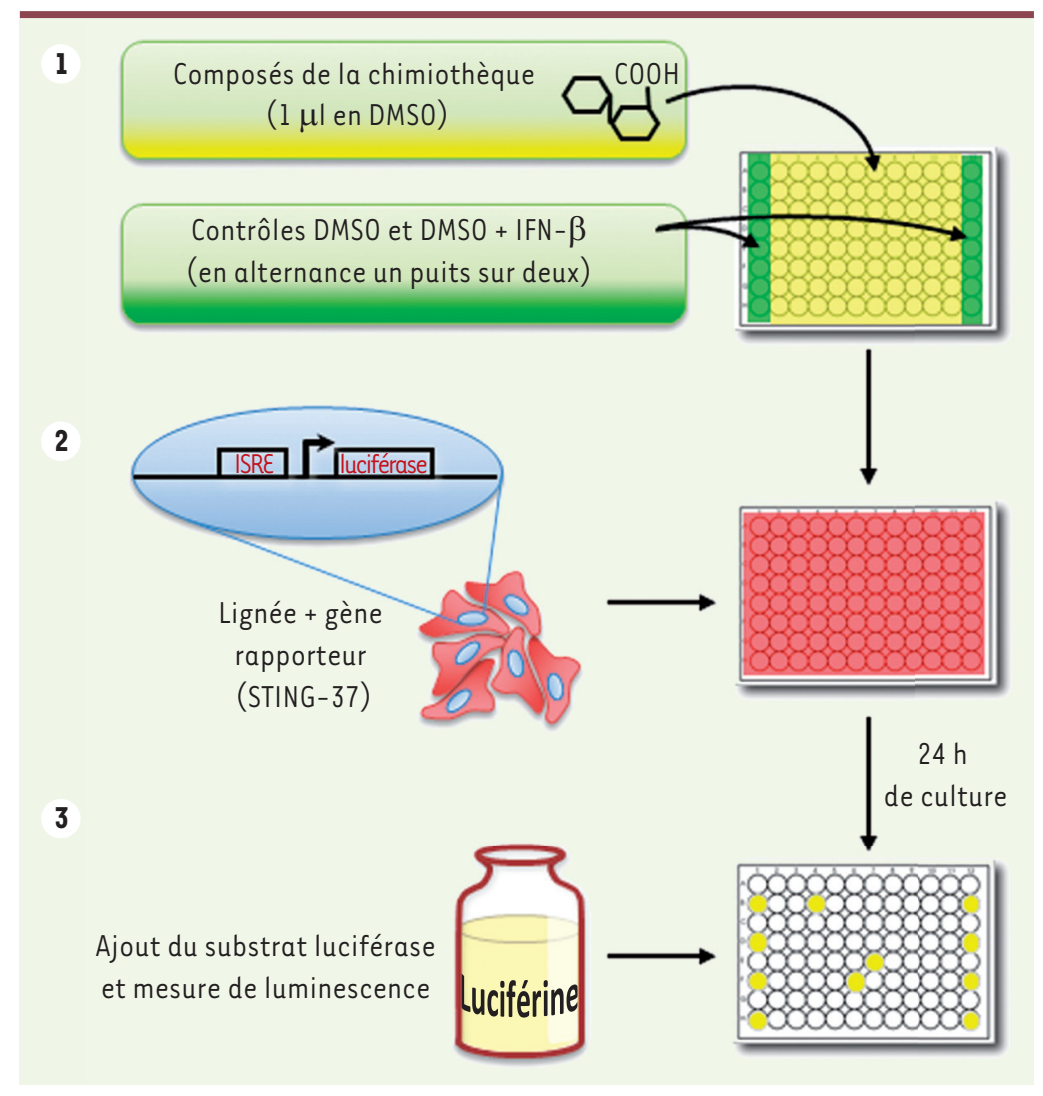

Figure 2. Schéma des différentes étapes du test phénotypique miniaturisé mis au point pour l'identification de composés capables de stimuler la réponse immunitaire innée antivirale. La première étape consiste en un dépôt des composés des chimiothèques disponibles (Tableau I) dans des plaques 96 puits (un composé par puits dans du DMSO). Les colonnes 1 et 12 sont dédiées aux contrôles (négatifs en présence de DMSO seul et positifs en présence d'IFN- $\beta$ ) : les valeurs obtenues pour ces contrôles serviront à calculer le facteur Z' [27], qui rend compte de la qualité et de la reproductibilité du test sur la base des écarttypes et des moyennes des minima (contrôles négatifs) et des maxima (contrôles positifs). Puis, des cellules transfectées de façon stable avec un plasmide portant la construction ISRE-luc (lignée cellulaire STING-37) sont ajoutées dans les puits des plaques ( $100 \mu \mathrm{l}$ par puits). Après $24 \mathrm{~h}$ d'incubation à $37^{\circ} \mathrm{C}$ en présence de $5 \%$ de $\mathrm{CO}_{2}$, le substrat de la luciférase est ajouté dans chaque puits $\left(50 \mu \mathrm{L}\right.$ Bright-Glo ${ }^{T M}$ luciferase assay system, Promega), et incubé à température ambiante 6 minutes. La mesure de luminescence est ensuite réalisée sur un lecteur de plaques multimode (temps d'intégration de $100 \mathrm{~ms}$ ).

avons développé un protocole de criblage en culture cellulaire basé sur l'utilisation de souches recombinantes des virus de la rougeole et du chikungunya, qui expriment la luciférase comme rapporteur de la réplication virale [12]. Ces deux virus étant très différents, aussi bien en termes de structure que de stratégie de réplication, nous avons montré qu'il était possible d'isoler, grâce à ce protocole, des composés ayant une activité antivirale à large spectre. Par ailleurs, plusieurs de ces molécules présentent une activité antivirale variable en fonction du type cellulaire considéré, ce qui suggère que leur cible est un facteur de la cellule hôte plutôt que la machinerie de réplication virale. Nous cherchons actuellement à déterminer leur mode d'action.

\section{Stimuler la réponse immunitaire innée des cellules}

Parallèlement à ce travail, nous avons développé un test phénotypique permettant de sélectionner des composés capables de stimuler la réponse immunitaire innée antivirale des cellules humaines, et notamment l'expression des gènes induits par les interférons de type I (IFN- $\alpha / \beta$ ) ou ISG (interferon-stimulated genes). Ces gènes codent plusieurs facteurs qui vont inhiber des étapes différentes du cycle viral, et permettre ainsi l'établissement d'un état de résistance aux infections virales [13]. Leur synthèse est déclenchée par la reconnaissance de motifs moléculaires associés aux organismes pathogènes (ou PAMP, pour pathogen-associated molecular patterns) par des récepteurs cellulaires appelés PRR (pattern recognition receptors) [14]. La détection de l'agent pathogène déclenche ensuite une cascade de signalisation entraînant notamment l'activation des facteurs de transcription IRF3 et 7 (interferon regulatory factor-3/7), tous deux impliqués dans l'activation directe de certains ISG et dans I'induction des IFN- $\alpha / \beta$. Comme la plupart des cytokines, les IFN- $\alpha / \beta$ exercent une action paracrine sur les cellules voisines et une action autocrine sur la cellule productrice elle-même. La fixation des IFN de type I sur leur récepteur IFNAR entraîne I'activation du facteur de transcription ISGF3 formé de STAT1, STAT2 et IRF9, et I'induction des ISG. L'élément de réponse à ISGF3 présent dans le promoteur des ISG est connu sous le nom d'ISRE (interferon-stimulated response element). Pour identifier de petites molécules capables de stimuler l'expression des ISG, nous avons utilisé des cellules humaines transfectées par un gène rapporteur exprimant la luciférase sous contrôle de I'élément de réponse ISRE (lignée cellulaire STING-37) (Figure 2) [15]. D’autres équipes ont développé une stratégie similaire [16-18]. Ainsi, l'induction de ce gène rapporteur par un composé se traduit par une augmentation de l'activité luciférase. En utilisant ce test, nous avons réalisé une campagne de criblage de la banque de molécules disponible sur la plateforme CBC. Nous avons identifié ainsi plusieurs inducteurs du gène rapporteur ISRE-luc, et ces composés ont ensuite été testés pour leur capacité à inhiber la réplication de différents virus, afin d'évaluer leur activité antivirale. 


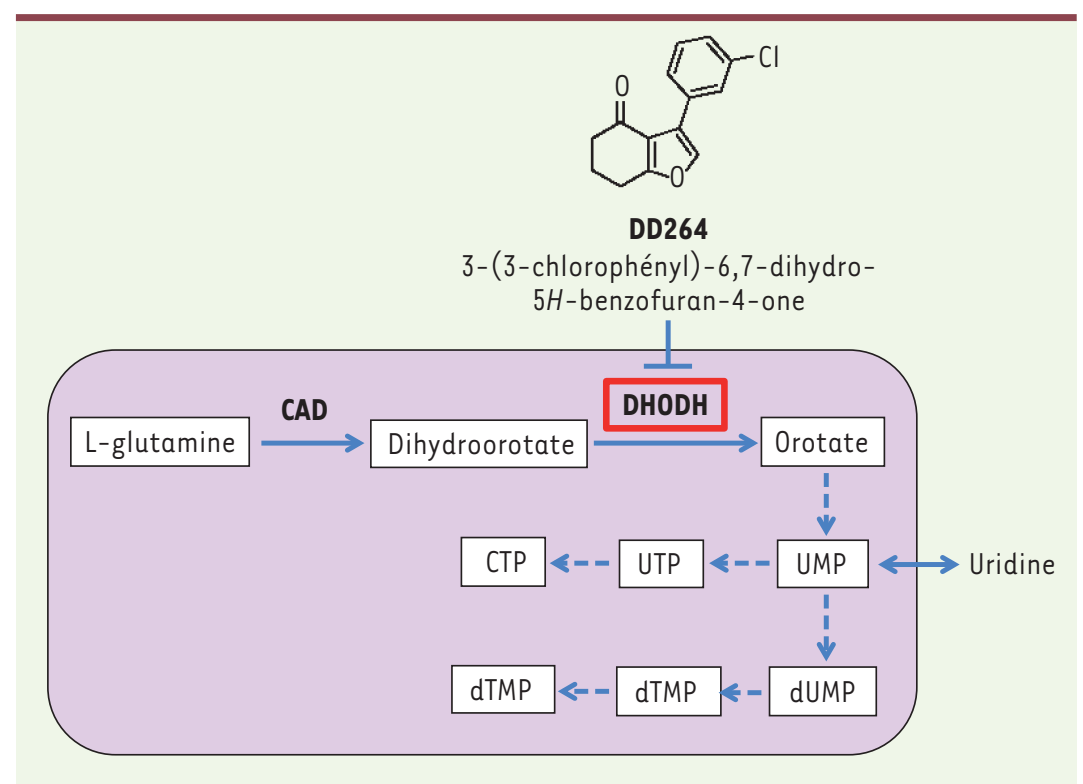

Figure 3. Représentation partielle de la biosynthèse des pyrimidines. Dans la voie de novo, les trois premières étapes sont réalisées au sein d'un complexe enzymatique multifonctionnel appelé le CAD (carbamyl phosphate synthétase - aspartate transcarbamylase - dihydroorotase) : à partir de glutamine est formé successivement le carbamoyl phosphate, puis le carbamoyl aspartate et enfin le dihydroorotate. La quatrième étape consiste en une déshydrogénation du dihydroorotate pour conduire à la formation d'orotate : I'enzyme responsable de cette réaction est la dihydroorotate déshydrogénase (DHODH). C'est cette enzyme qui est inhibée par le composé DD264. Les deux étapes suivantes de cette biosynthèse conduisent à la formation d'UMP, et sont à nouveau réalisées au sein d'un complexe multifonctionnel chez les mammifères (UMP synthétase). L'UMP sert de base pour l'obtention des autres nucléotides pyrimidiques. La voie de sauvetage est la deuxième source de nucléotides pyrimidiques. Au cours de cette voie, ces derniers sont synthétisés à partir des intermédiaires (les bases puriques et les nucléosides) du catabolisme (dégradation) des nucléotides. L'uracile, puis l'uridine, sont récupérés pour former l'UMP (par l'uridine phosphorylase puis l'uridine kinase).

Parmi ces molécules, le composé DD264 (Figure 3), issu de la chimiothèque de l'Institut Curie, a fait l'objet d'une étude détaillée [15]. Ce composé est un faible inducteur du gène rapporteur ISRE-luc, et donc a priori un mauvais stimulateur des ISG et de la réponse cellulaire innée antivirale. Pourtant, nous avons constaté qu'in vitro, la molécule DD264 inhibe très efficacement la réplication de différents virus à génome ARN de polarité positive (virus du chikungunya, virus du Nil occidental, virus de la dengue, coronavirus 229ع, coxsackievirus B3) ou négative (virus de la rougeole, virus respiratoire syncytial, virus parainfluenza de type 3 ). L'activité originale de ce composé nous a conduits à étudier son mécanisme d'action et à rechercher sa cible.

\section{Caractérisation et mode d'action du composé à activité antivirale DD264}

\section{DD264 inhibe la biosynthèse des pyrimidines}

Dans un premier temps, nous avons étudié le transcriptome de lignées cellulaires humaines traitées par la molécule DD264, par les techniques de PCR quantitative ou d'hybridation des transcrits sur des puces à ADN. Les résultats obtenus ont permis de montrer que cette molécule n'induit qu'un ensemble restreint de gènes antiviraux sous contrôle d'un élément de réponse ISRE, et cela de façon très peu efficace, ce qui est surprenant étant donné la forte activité antivirale du DD264. En fait, le composé DD264 est capable d'amplifier la réponse cellulaire antivirale ce qui peut expliquer son activité antivirale à large spectre. En effet, la molécule DD264 augmente l'expression du gène rapporteur ISRE-luc et des gènes codant les ISG de cellules préalablement stimulées avec I'IFN- $\beta$, ou transfectées par des ARN synthétiques qui miment une infection virale et stimulent les PRR. Cependant, ces résultats ne nous donnaient aucune indication sur la cible du DD264, laquelle restait à identifier.
Alors que nous testions sans succès différentes hypothèses, plusieurs groupes ont publié simultanément des travaux montrant que des molécules inhibitrices de la voie de biosynthèse des pyrimidines sont des antiviraux à large spectre [19-22]. Même si rien ne suggérait un lien fonctionnel entre cette voie métabolique et la réponse innée antivirale, nous avons testé la capacité du composé DD264 à bloquer la biosynthèse de novo des pyrimidines (Figure 3 ). Cette voie métabolique assure la synthèse d'uridine 5'-monophosphate (UMP), lequel sert de précurseur pour les autres nucléotides pyrimidiques nécessaires à la cellule. L'autre source de synthèse de ces nucléotides provient de la voie dite de sauvetage (ou catabolisme). Bien que cette dernière soit suffisante dans des cellules quiescentes, la voie de novo est indispensable pour toute cellule en division ou métaboliquement active. En mesurant les nucléotides présents dans des cellules traitées ou non avec le DD264, nous avons constaté que ce composé entraîne un effondrement du pool de pyrimidines. Par ailleurs, en supplémentant le milieu de culture avec de I'uridine, il est possible de restaurer la réplication virale dans des cellules traitées avec le composé DD264. Ces résultats montrent que le composé DD264 est effectivement un inhibiteur des premières étapes de cette voie de biosynthèse (Figure 3 ), plus particulièrement de la dihydroorotate déshydrogénase (DHODH). Cette enzyme de la membrane mitochondriale interne chez les eucaryotes assure la conversion du dihydroorotate en orotate en utilisant comme cofacteurs la FMN (flavine 
mononucléotide) et l'ubiquinone [23]. En utilisant un test enzymatique, nous avons démontré que le composé DD264 inhibe directement l'activité de la DHODH. Par ailleurs, l'addition d'orotate (produit de la réaction catalysée par la DHODH) au milieu de culture est capable de contrecarrer - comme l'uridine - les effets antiviraux de cette molécule, alors que le dihydroorotate (substrat de la DHODH) est sans effet. L'ensemble de ces résultats ont permis d'identifier la DHODH comme la cible principale du DD264.

\section{Effet antiviral du DD264 : une action concertée entre ISG et métabolisme des pyrimidines}

Quel est alors le lien entre biosynthèse des pyrimidines et réponse innée antivirale? Après avoir identifié la DHODH comme cible du composé DD264, nous avons montré que la chute du pool de pyrimidines dans les cellules traitées est directement responsable de l'amplification de la réponse innée antivirale. En effet, dès lors que le milieu de culture est supplémenté en uridine, la capacité du composé DD264 à amplifier le niveau d'expression des ISG disparaît. Nous ignorons encore la voie de signalisation qui relie ainsi l'équilibre du pool de pyrimidines à la réponse innée antivirale. Néanmoins, l'expression des ISG semble jouer un rôle important dans l'activité antivirale du DD264 et du brequinar, un autre inhibiteur de la DHODH développé comme anticancéreux à la fin des années 1980 [24]. En effet, un inhibiteur de I'ARN polymérase II, I' $\alpha$-amanitine, annule l'effet antiviral du DD264 et du brequinar. La leptomycine-B, qui bloque l'export des ARNm du noyau vers le cytoplasme, bloque aussi l'action antivirale du DD264. Enfin, nous avons montré que l'expression des ISG dans les cellules HEK-293T est dépendante du facteur de transcription IRFl. Lorsque l'expression de ce dernier est supprimée par siARN (silencing RNA), l'effet antiviral du DD264 ou du brequinar est fortement diminué. En réponse au blocage de la voie de biosynthèse des pyrimidines, les ISG joueraient donc un rôle important dans le blocage de la réplication virale. Ces résultats suggèrent que l'effet antiviral de ces inhibiteurs n'est pas uniquement la conséquence directe d'une chute du pool de pyrimidines dans la cellule, mais implique également l'induction d'un stress cellulaire, l'expression des ISG et l'amplification de la réponse innée antivirale [15].

\section{Quel avenir thérapeutique pour les inhibiteurs}

de la biosynthèse des pyrimidines ?

Quel est l'avenir du composé DD264 dans le traitement des infections virales? La concentration inhibitrice médiane de cette molécule $\left(I_{50}\right)$ est d'environ $16 \mu \mathrm{M}$, ce qui est encore trop élevé pour envisager son utilisation in vivo. Afin d'augmenter l'activité de ce composé, environ 80 analogues ont été synthétisés mais aucun n'a montré pour l'instant une $\mathrm{IC}_{50}$ significativement plus faible. Cependant, nous avons récemment identifié par la même stratégie d'autres composés inhibiteurs de la voie de biosynthèse des pyrimidines et qui sont mille fois plus actifs. Nos efforts se concentrent actuellement sur ces nouvelles séries chimiques. L'originalité de nos travaux réside dans le test phénotypique qui nous a permis d'identifier le DD264 et le lien que nous avons ainsi établi entre la voie de biosynthèse des pyrimidines et la réponse innée antivirale. Cependant, plusieurs groupes qui cherchaient à identifier directement des composés antiviraux dans des tests mesurant la réplication d'un virus en culture ont également isolé des inhibiteurs de cette voie métabolique, et qui ciblent notamment la DHODH [19-22, 25]. Les essais réalisés in vivo chez la souris par ces différentes équipes ont donné des résultats mitigés. Si la réplication du cytomégalovirus est efficacement bloquée par ce type de drogue [26], aucun effet inhibiteur n'a pu être établi pour le virus de la dengue, de la grippe ou le virus respiratoire syncytial humain $[19,20,22]$. En effet, le taux plasmatique d'uridine est relativement élevé, ce qui permet à de nombreuses cellules de l'organisme de s'affranchir de la voie de biosynthèse de novo des pyrimidines. Par ailleurs, les cellules quiescentes de l'organisme sont peu actives sur le plan métabolique, ce qui limite également l'utilisation d'inhibiteurs de la voie de biosynthèse des pyrimidines pour induire un effet antiviral. Cependant, ces composés pourraient s'avérer efficaces dans le traitement d'infections virales affectant plus spécifiquement les cellules ayant un fort taux de division et de renouvellement (cellules épithéliales des muqueuses, lymphocytes activés, etc.). Des études complémentaires seront nécessaires pour répondre à cette question.

Enfin, il faut rappeler que différents antimétabolites sont utilisés avec succès en cancérologie, dans le traitement des maladies auto-immunes et dans la prévention des rejets de greffes. À ce titre, le tériflunomide (Aubagio), et sa prodrogue apparentée, le léflunomide (Arava), sont prescrits dans le traitement de l'arthrite rhumatoïde et de la sclérose en plaques. Ces drogues sont des inhibiteurs de la DHODH, mais leur effet antiprolifératif sur les lymphocytes serait également lié à une action parallèle sur différentes kinases cellulaires. Ceci suggère que certains des nombreux inhibiteurs de la DHODH récemment isolés pour leur activité antivirale pourraient être développés à l'avenir dans le traitement de pathologies sans lien direct avec les infections virales.

Ainsi, l'identification du composé DD264 par criblage phénotypique, puis la découverte de son mode action, ont réservé de nombreuses surprises avec un cheminement inattendu de la réponse antivirale innée au métabolisme des nucléosides. Ces travaux démontrent l'intérêt des tests fonctionnels sur cellules. Ces derniers permettent non seulement d'identifier des molécules actives capables de moduler un mécanisme cible, mais aussi de mettre en évidence des processus biologiques originaux. De ce point de vue, les composés chimiques perturbent le système biologique étudié, au même titre qu'une mutation génétique ou un siARN. Les 
chimiothèques sont alors employées comme un ensemble de «perturbagènes » permettant de sonder le fonctionnement du vivant. $\diamond$

\section{SUMMARY}

Stimulation of the antiviral innate immune response by pyrimidine biosynthesis inhibitors: a surprise of phenotypic screening RNA viruses are responsible for major human diseases such as flu, bronchitis, dengue, hepatitis $C$ or measles. They also represent an emerging threat because of increased worldwide exchanges and human populations penetrating more and more natural ecosystems. Recent progresses in our understanding of cellular pathways controlling viral replication suggest that compounds targeting host cell functions, rather than the virus itself, could inhibit a large panel of RNA viruses. In particular, several academic laboratories and private companies are now seeking molecules that stimulate the host innate antiviral response. One appealing strategy is to identify molecules that induce the large cluster of antiviral genes known as Interferon-Stimulated Genes (ISGs). To reach this goal, we have developed a phenotypic assay based on human cells transfected with a luciferase reporter gene under control of an interferon-stimulated response element (ISRE). This system was used in a high-throughput screening of chemical libraries comprising around 54,000 compounds. Among validated hits, compound DD264 was shown to boost the innate immune response in cell cultures, and displayed a broad-spectrum antiviral activity. While deciphering its mode of action, DD264 was found to target the fourth enzyme of de novo pyrimidine biosynthesis, namely the dihydroorotate dehydrogenase (DHODH). Thus, our data unraveled a yet unsuspected link between pyrimidine biosynthesis and the innate antiviral response. $\diamond$

\section{LIENS D'INTÉRÊT}

Les auteurs déclarent n'avoir aucun lien d'intérêt concernant les données publiées dans cet article.

\section{RÉFÉRENCES}

1. Debing $Y$, Jochmans $D$, Neyts J. Intervention strategies for emerging viruses: use of antivirals. Curr Opin Virol $2013 ; 3: 217-24$.

2. Antona D, Baudon C, Freymuth F, et al. La rougeole en France. Med Sci (Paris) 2012 ; 28 : 1003-7.

3. Nair H, Nokes DJ, Gessner BD, et al. Global burden of acute lower respiratory infections due to respiratory syncytial virus in young children: a systematic review and meta-analysis. Lancet $2010 ; 375: 1545-55$.

4. Graham RL, Donaldson EF, Baric RS. A decade after SARS: strategies for controlling emerging coronaviruses. Nat Rev Microbiol $2013 ; 11: 836-48$.

5. International ASSWGoHIVC, Deeks SG, Autran B, et al. Towards an HIV cure: a global scientific strategy. Nat Rev Immunol $2012 ; 12: 607-14$.

6. Pockros PJ. Advances in newly developing therapy for chronic hepatitis C virus infection. Front Med $2014 ; 8: 166-74$.
7. Pol S. Virus de l'hépatite C. 25 ans, la fin de l'histoire? Med Sci (Paris) 2013 ; 29 : $998-1003$.

8. Warren TK, Wells J, Panchal RG, et al. Protection against filovirus diseases by a novel broad-spectrum nucleoside analogue BCX4430. Nature $2014 ; 508$ : 402-5.

9. Ison MG. Antivirals and resistance: influenza virus. Curr Opin Virol $2011 ; 1$ : 563-73.

10. Prussia A, Thepchatri P, Snyder JP, Plemper RK. Systematic approaches towards the development of host-directed antiviral therapeutics. Int J Mol Sci $2011 ; 12: 4027-52$.

11. Es-Saad S, Tremblay N, Baril M, Lamarre D. Regulators of innate immunity as novel targets for panviral therapeutics. Curr Opin Virol $2012 ; 2: 622-8$.

12. Lucas-Hourani M, Munier-Lehmann $\mathrm{H}$, Helynck 0 , et al. High-throughput screening for broad-spectrum chemical inhibitors of RNA viruses. J Vis Exp $2014 ; 87$ : e51222.

13. MacMicking JD. Interferon-inducible effector mechanisms in cellautonomous immunity. Nat Rev Immunol 2012 ; 12 : 367-82.

14. Thompson MR, Kaminski JJ, Kurt-Jones $\varepsilon A$, Fitzgerald KA. Pattern recognition receptors and the innate immune response to viral infection. Viruses 2011 ; $3: 920-40$.

15. Lucas-Hourani M, Dauzonne D, Jorda $P$, et al. Inhibition of pyrimidine biosynthesis pathway suppresses viral growth through innate immunity. PLoS Pathog $2013 ; 9$ : el003678.

16. Bedard KM, Wang ML, Proll SC, et al. Isoflavone agonists of IRF-3 dependent signaling have antiviral activity against RNA viruses. J Virol $2012 ; 86$ : 7334-44.

17. Tai ZF, Zhang GL, Wang F. Identification of small molecule activators of the janus kinase/signal transducer and activator of transcription pathway using a cell-based screen. Biol Pharm Bull 2012 ; 35 : 65-71.

18. Patel DA, Patel AC, Nolan WC, et al. High throughput screening for small molecule enhancers of the interferon signaling pathway to drive nextgeneration antiviral drug discovery. PLoS One $2012 ; 7$ : e36594.

19. Bonavia A, Franti M, Pusateri Keaney $\varepsilon$, et al. Identification of broadspectrum antiviral compounds and assessment of the druggability of their target for efficacy against respiratory syncytial virus (RSV). Proc Natl Acad Sci USA $2011 ; 108$ : 6739-44.

20. Wang QY, Bushell S, Qing M, et al. Inhibition of dengue virus through suppression of host pyrimidine biosynthesis. J Virol $2011 ; 85$ : 6548-56.

21. Hoffmann HH, Kunz A, Simon VA, et al. Broad-spectrum antiviral that interferes with de novo pyrimidine biosynthesis. Proc Natl Acad Sci USA $2011 ; 108: 5777-82$.

22. Smee DF, Hurst BL, Day CW. D282, a non-nucleoside inhibitor of influenza virus infection that interferes with de novo pyrimidine biosynthesis. Antivir Chem Chemother $2012 ; 22: 263-72$.

23. Munier-Lehmann H, Vidalain PO, Tangy F, Janin YL. On dihydroorotate dehydrogenases and their inhibitors and uses. J Med Chem $2013 ; 56: 3148-67$.

24. Liu S, Neidhardt $\varepsilon A$, Grossman TH, et al. Structures of human dihydroorotate dehydrogenase in complex with antiproliferative agents. Structure $2000 ; 8$ : 25-33.

25. Ortiz-Riano $\varepsilon$, Ngo $N$, Devito $S$, et al. Inhibition of arenavirus by $A 3$, a pyrimidine biosynthesis inhibitor. J Virol $2014 ; 88$ : 878-89.

26. Marschall M, Niemann I, Kosulin K, et al. Assessment of drug candidates for broad-spectrum antiviral therapy targeting cellular pyrimidine biosynthesis. Antiviral Res $2013 ; 100: 640-8$.

27. Zhang JH, Chung TD, Oldenburg KR. A simple statistical parameter for use in evaluation and validation of high throughput screening Assays. J Biomol Screen 1999; $4: 67-73$

\section{TIRÉS À PART}

H. Munier-Lehmann

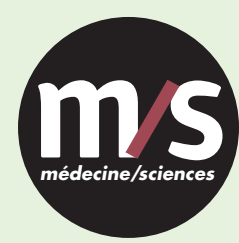

Tarifs d'abonnement $m / s-2015$

Abonnez-vous

à médecine/sciences
$>$ Grâce à $m / s$, vivez en direct les progrès des sciences biologiques et médicales

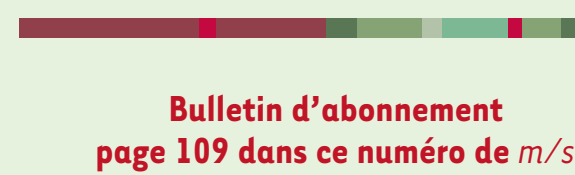

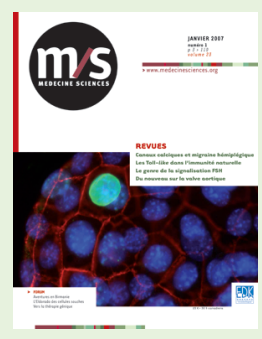

